\title{
ARTICLE
}

Clinical Study

\section{Improved relapse-free survival on aromatase inhibitors in breast cancer is associated with interaction between oestrogen receptor- $\alpha$ and progesterone receptor-b}

\author{
Cameron E. Snell $\mathbb{I}^{1,2}$, Madeline Gough ${ }^{1,2}$, Cheng Liu $^{2}$, Kathryn Middleton ${ }^{3,5}$, Christopher Pyke ${ }^{4,5}$, Catherine Shannon ${ }^{3,5}$, \\ Natasha Woodward ${ }^{3,5}$, Theresa E. Hickey ${ }^{6}$, Jane E. Armes ${ }^{2}$ and Wayne D. Tilley ${ }^{6}$
}

\begin{abstract}
BACKGROUND: Recent pre-clinical studies indicate that activated progesterone receptor (PR) (particularly the PR-B isoform) binds to oestrogen receptor-a (ER) and reprogrammes transcription toward better breast cancer outcomes. We investigated whether ER and PR-B interactions were present in breast tumours and associated with clinical parameters including response to aromatase inhibitors.

METHODS: We developed a proximity ligation assay to detect ER and PR-B (ER:PR-B) interactions in formalin-fixed paraffinembedded tissues. The assay was validated in a cell line and patient-derived breast cancer explants and applied to a cohort of 229 patients with ER-positive and HER2-negative breast cancer with axillary nodal disease.

RESULTS: Higher frequency of ER:PR-B interaction correlated with increasing patient age, lower tumour grade and mitotic index. A low frequency of ER:PR-B interaction was associated with higher risk of relapse. In multivariate analysis, ER:PR-B interaction frequency was an independent predictive factor for relapse, whereas PR expression was not. In subset analysis, low frequency of ER: PR-B interaction was predictive of relapse on adjuvant aromatase inhibitor (HR 4.831, $p=0.001$ ), but not on tamoxifen (HR 1.043, $p=0.939$ ).
\end{abstract}

CONCLUSIONS: This study demonstrates that ER:PR-B interactions have utility in predicting patient response to adjuvant AI therapy.

British Journal of Cancer (2018) 119:1316-1325; https://doi.org/10.1038/s41416-018-0331-3

\section{BACKGROUND}

Approximately $80 \%$ of breast cancers express oestrogen receptor$a(E R)^{1,2}$ and are considered to be driven by the trophic effects of oestrogen. Expression of ER by immunohistochemistry remains the only clinical biomarker predictive of benefit to adjuvant antioestrogen therapies, which include two broad classes of drugs; non-steroidal aromatase inhibitors (Als) and the selective ER modulator, tamoxifen. Progesterone receptors ( $P R$, comprising $A$ and $B$ isoforms) are upregulated in response to ER signalling in normal and malignant breast tissues. ${ }^{3}$ Antibodies used to detect PR for clinical and investigative purposes largely detect both PR isoforms and using these, PR has been established as a biomarker of good prognosis in breast cancer. ${ }^{4}$ Higher levels of PR expression are associated with a good response to tamoxifen, ${ }^{4,5}$ which until the development of aromatase inhibitors (Als) was the major firstline adjuvant endocrine therapy for all cases of ER-positive $(E R+)$ breast cancer. Currently, tamoxifen is mainly prescribed to premenopausal women and Als to postmenopausal women, with some exceptions. ${ }^{6-8}$ In general, Als may confer a survival advantage compared to tamoxifen. ${ }^{9}$ However, PR expression does not predict therapeutic benefit of Als. ${ }^{6-8}$

Pre-clinical studies have shown that ER and PR form a physical interaction in the presence of their cognate hormones and that this activity may promote better disease outcomes. ${ }^{10-14}$ In the presence of oestrogen and a progestogen, including endogenous progesterone (P4), PR alters the interaction between ER and chromatin to change the transcriptional output of ER+ breast cancer cells. ${ }^{12-14}$ Ligand-activated PR redirects ER chromatin binding to sites enriched for progesterone response motifs ${ }^{12}$ and distal enhancers enriched for BRCA1 motifs. ${ }^{13}$ Moreover, expression of a gene signature associated with PR-mediated reprogramming of ER binding is associated with good prognosis in primary breast cancer cohorts. ${ }^{12}$ Consistent with these findings, progestogen treatment inhibits oestrogen-dependent growth in various preclinical models of breast cancer (e.g. breast cancer cell

\footnotetext{
${ }^{1}$ Cancer Pathology Research Group, Mater Research Institute-The University of Queensland, Translational Research Institute, Woolloongabba, QLD 4102, Australia; ${ }^{2}$ Department

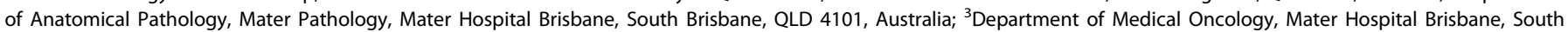

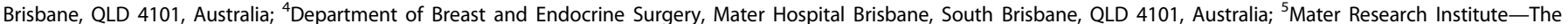

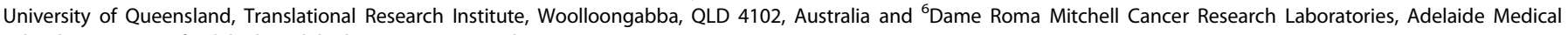
School, University of Adelaide, Adelaide, SA 5000, Australia

Correspondence: Cameron E. Snell (cameron.snell@gmail.com)

These authors contributed equally: Jane E. Armes, Wayne D. Tilley.
}

Received: 15 May 2018 Revised: 14 October 2018 Accepted: 20 October 2018

Published online: 9 November 2018 
lines, ex-vivo culture of clinical breast cancer tissues and patientderived xenografts). ${ }^{12-14}$

Two progesterone receptor isoforms, PR-A and PR-B, are transcribed from a single gene, the PGR. ${ }^{15}$ The two isoforms are identical apart from an additional 165 amino acids present in the $\mathrm{N}$-terminus of PR-B. In the presence of both ER and PR agonist ligands, immunoprecipitation of ER shows a specific increase in PR-B interaction in the ER+, PR+ breast cancer cell line T47D. ${ }^{13}$ However, in the presence of activated ER, unliganded PR promotes expression of a subset of ER target genes and enhances proliferation, ${ }^{11}$ highlighting the importance of ligand activation of PR. Up to $29 \%$ of ER+ breast cancers have a heterozygous or homozygous deletion of PGR, which occurs more often in the luminal $B$ subtype of breast cancers. ${ }^{12}$ Therefore, it is not surprising that the luminal B breast cancer subtype is associated with a higher proliferation rate and poorer prognosis than luminal A cancers. ${ }^{16}$ Loss of PGR is a mechanism by which ER+ tumours may evade the antagonistic effect of PR signalling on ER-mediated oncogenesis. In support of this, loss of PGR in ER+ cancers is associated with poor prognosis. ${ }^{12}$ In ER+PR+ tumours, lack of adequate PR activation could also feasibly be a cause of unrestrained ER activity.

The ratio of PR-A to PR-B has been investigated by immunoblot analysis and varies between breast cancers. ${ }^{17-20}$ Patients with PR+ tumours that have a lower proportion of PR-B have a worse prognosis and are more likely to relapse on tamoxifen, ${ }^{18}$ while tumours with a higher proportion of PR-A responded to the antiprogesterone mifepristone in ex vivo models. ${ }^{19}$ Patients whose tumours expressed a gene signature associated with a high PR-A to $P R-B$ ratio also have a poorer survival outcome. ${ }^{21}$ The two isoforms differentially reprogramme ER-binding: PR-B predominantly acts to redistribute ER genomic recruitment while PR-A predominantly inhibits ER chromatin binding. ${ }^{21}$ In T47D cells engineered to express a single isoform, only PR-B decreased oestrogen-induced invasion. ${ }^{21}$ These findings suggest PRmediated reprogramming of ER is dependent on PR isoformspecific expression.

Oestrogen is present at sufficient levels in post-menopausal women to promote ER+ breast cancer and these patients benefit from treatment with aromatase inhibitors. ${ }^{7}$ The majority of oestrogen production in postmenopausal women occurs in peripheral tissues that express aromatase, including the breast. ${ }^{22}$ Since circulating progesterone is present at very low levels in postmenopausal women, ${ }^{23}$ exogenous treatment with a PR agonist may be a therapeutic strategy to benefit patients with $\mathrm{ER}+$ breast cancer by exploiting cross-talk between ER and PR. ${ }^{24}$ In advanced ER+ breast cancer, trials of progestins such as megesterol acetate or medroxyprogesterone acetate have consistently shown significant clinical benefit, including in women who had previously relapsed on either an $\mathrm{Al}^{25}$ or tamoxifen. ${ }^{26,27}$ Clinical trials are underway to test efficacy of progestogens in the neoadjuvant setting. ${ }^{28}$ Although the abovementioned studies propose that induction of ER:PR-B interaction would be therapeutically beneficial, such interactions have not yet been shown to occur in clinical specimens. The aim of our study was to validate a proximity ligation assay (PLA) to detect an interaction between ER and PR-B (ER:PR-B) in formalin-fixed, paraffin embedded (FFPE) tissues and investigate whether this interaction was predictive of relapse in a cohort of women with ER+ breast cancer treated with adjuvant endocrine therapy.

\section{METHODS}

Cell culture

The T47D breast cancer cell line was acquired from the ATCC and cultured in DMEM supplemented with $10 \%$ FBS. The cells were regularly tested for mycoplasma infection. To stimulate ER and PR interactions, the cells were first cultured in phenol red-free DMEM supplemented with charcoal stripped FBS (Gibco, no. 12676011) for $48 \mathrm{~h}$, then the media was supplemented with vehicle (ethanol), oestradiol $\left(E_{2}\right)$ (Sigma, no. E2758), progesterone (Sigma, no. P6149) or the combination of $E_{2}$ and progesterone both at a final concentration of $10 \mathrm{nM}$ for $24 \mathrm{~h}$. Cells were mechanically lifted, fixed in $10 \%$ neutral buffer formalin for $24 \mathrm{~h}$ then resuspended in $2 \%$ molten agarose dissolved in $10 \%$ formalin. The cells and agarose suspension were centrifuged to form a pellet. Cell pellets were processed in tissue cassettes at Mater Pathology as per clinical specimens.

\section{Patient-derived tumour explants (PDEs)}

Tumour samples were obtained following informed consent from women undergoing surgery for breast cancer at the Burnside War Memorial Hospital, Adelaide. This study was approved by the University of Adelaide Human Research Ethics Committee (approval numbers: H-065-2005; H-169-2011). Excised tissue samples were cultured ex vivo as previously described. ${ }^{12,29}$ Briefly, PDEs were cultured on gelatine sponges for $36 \mathrm{~h}$ then treated with the following conditions: vehicle (ethanol), $E_{2}(10 \mathrm{nM})$, a synthetic progestin R5020 (10 nM) or the combination of $E_{2}$ and R5020 (both at $10 \mathrm{nM}$ ) with treatment for $48 \mathrm{~h}$. Explants were fixed in $10 \%$ neutral buffered formalin overnight and processed as per clinical specimens.

\section{Proximity-ligation assays}

The proximity-ligation assay (PLA) can be used to detect proteins, interactions and modifications with high sensitivity and specificity. ${ }^{30}$ PLA requires protein recognition by pairs of antibody conjugates and improves specificity of protein detection over immunohistochemical assays. ${ }^{31}$ FFPE tissues were sectioned at 6 $\mu \mathrm{M}$, deparaffinised and antigen retrieved in citrate buffer at $\mathrm{pH} 6$ using a Decloaking Chamber (Biocare Medical). Sections were blocked, and primary antibodies were diluted in antibody diluent (Roche, no. 251-018) and incubated overnight at $4{ }^{\circ} \mathrm{C}$. To detect ER and PR-B interactions, antibodies from two different species were used; monoclonal rabbit anti-ER (Thermo Scientific, clone SP1) and monoclonal mouse anti-PR (Sigma, clone 3E11 - raised against an immunogen specific to the amino-terminus of the PR-B isoform), both used at 1:100 dilution. For expression of PR-B, the same mouse anti-PR-B was incubated with rabbit anti-PR (Ventana, clone $1 E 2$, detecting both $P R A$ and $B$ isoforms ${ }^{32}$ ). This was followed by incubation with the PLA-probes Duolink in Situ PLA Probe Anti-Mouse PLUS (Sigma, no. DUO92001-100RXN) and AntiRabbit MINUS (Sigma, no. DUO92005-100RXN) for $60 \mathrm{~min}$ at $37^{\circ} \mathrm{C}$ in a pre-heated humidity chamber. Ligation took place for $30 \mathrm{~min}$ and amplification for $120 \mathrm{~min}$ at $37^{\circ} \mathrm{C}$ using Duolink in Situ Detection reagents brightfield (Sigma, no. DUO92012-100RXN). To detect the rolling circle amplification product, horse radish peroxidase-conjugated probe was incubated for $60 \mathrm{~min}$ at room temperature and substrate solution was applied for $10 \mathrm{~min}$ also at room temperature. Slides were counterstained with haematoxylin. Staining was independently scored by two breast histopathologists (CS, CL) by counting the number of signals per nucleus in 20 cells in the areas of tumour with greatest numbers of signals, a similar method to that used to score HER2 detected by in situ hybridisation assays. Scores were averaged to determine a final score. The interactions detected by this assay are referred to as "ER:PR-B" and the signals detected by the PR-B PLA are referred to as "PR-B".

Immunohistochemistry

ER and PR immunohistochemistry was performed with anti-ER (Ventana, clone SP1) and anti-PR (Ventana, clone 1E2) using the Ventana BenchMark ULTRA automated slide stainer (Roche). The Ventana anti-PR antibody clone 1E2 used widely by diagnostic pathology laboratories recognises both the PR A and B isoforms. ${ }^{32}$ ER and PR immunohistochemistry was scored by two breast 
histopathologists (CS, CL) using the 'Allred score' on a scale of $0-8 .^{33}$ In brief, the proportion of positive cells was evaluated as $0=$ no positive cells, $1=0-<1 \%$ positive cells, $2=1-<10 \%$ positive cells, $3=10-<33 \%$ positive cells, $4=33-<66 \%$ positive cells and $5=>66 \%$ positive cells. Additionally, the average intensity of staining was scored as $0=$ negative, $1=$ weak, $2=$ moderate or $3=$ strong. The intensity and proportion scores were added to obtain the 'Allred score'. A cut-off of $>2$ was considered positive (weak positive staining in $>1 \%$ of tumour cell nuclei) which is the cut-off used clinically. ${ }^{34}$

\section{Study population}

A cohort comprising a consecutive series of 229 patients who had surgery with curative intent for $\mathrm{ER}+$, human epidermal growth factor receptor 2-negative (HER2-negative) node-positive breast cancer (supplementary material Table S1) was analysed. All patients had lymph node metastatic deposits of at least $2.0 \mathrm{~mm}$ in size resected with curative intent (at least N1, all patients were stage II and $\mathrm{II}^{35}$ ). Patients had their tumours resected at the Mater Hospital Brisbane between January 2005 and December 2014. No patients had endocrine therapy prior to surgery. HER2 negativity was defined by negative immunohistochemistry and lacking amplification by in situ hybridisation of the ERBB2 gene. Recommendations for adjuvant treatment were made at the breast multidisciplinary meeting according to international guidelines and treatment decisions were made by patients in conjunction with their treating specialists. The median age of patients was 54 years at resection (range 27-88 years) and $71.2 \%$ were postmenopausal. $86.0 \%$ of patients had adjuvant chemotherapy, $81.7 \%$ adjuvant radiation and $94.8 \%$ adjuvant endocrine therapy. Of those that had endocrine therapy, $69.6 \%$ were treated with an aromatase inhibitor and $29.0 \%$ with tamoxifen. The median follow-up time was 5.1 years (range 0.9-11.3 years). Relapse was defined as either clinically or radiologically detected locoregional or distant metastatic disease. Relapse occurred in 48 patients $(21 \%)$ and the mean estimated relapse-free survival time was 8.8 years (SD 3.7 years). All patients were recommended adjuvant endocrine therapy post-surgery and patients were considered not to have taken adjuvant endocrine therapy if they took a total of $<2$ months treatment. The use of clinical information and tumour blocks was approved by the Mater Health Services Human Research Ethics Committee (approval number: HREC/15/MHS/123). Cores of the primary tumour from each patient were assembled into tissue microarrays (four cores per patient, each measuring $1.0 \mathrm{~mm}$ in diameter) ${ }^{36}$ using a semiautomated arrayer (Beecher Instruments). Four $1.0 \mathrm{~mm}$ cores have previously demonstrated spatial heterogeneity for ER in only $2 \%$ of cases and PR in $7 \%$ of cases. ${ }^{37}$ The study was designed to meet the REMARK guidelines for reporting tumour marker prognostic studies. $^{38}$

\section{Statistical design and analysis}

Statistical analysis was performed using SPSS V.22.0 (IBM) and GraphPad Prism V.7.03 (GraphPad Software, Inc). Correlations between ER:PR-B interactions, PR-B expression and clinical and pathological factors were determined using the 2-tailed Spearman's rank correlation coefficient $(r)$ as ER:PR-B interactions were not normally distributed. The Mann-Whitney $U$ test was used to compare test whether number of ER:PR-B interactions differed between PR - and PR + groups. Receiver operating characteristic (ROC) curves were used to determine the optimum cut-off of signals per cell with respect to relapse. Relapse-free survival analyses were carried out using Kaplan-Meier curves and significance determined by log-rank test. Univariate and multivariate Cox regression analyses were used to determine significant dependent and independent variables. Factors significant in the univariate analysis were included in the multivariate analysis. Associations for $2 \times 2$ tables were carried out using a Fisher's exact test, due to small numbers in some subgroups. The $3 \times 2$ table for association of tumour histologic type was tested for significance using a Fisher's exact test. All other $3 \times 2$ tables and $4 \times 2$ tables were tested for significance using the Cochran-Armitage test for trend, due to the variables being ordinal.

\section{RESULTS}

Visualisation of ER and PR interactions in FFPE tissue

We applied an ER:PR-B proximity ligation assay (PLA) to three $\mathrm{ER}+, \mathrm{PR}+\mathrm{PDEs}$ treated with vehicle, $\mathrm{E}_{2}$, the synthetic progestin R5020 or the combination of $E_{2}$ and R5020. An interaction between ER and PR-B was observed in all three of the untreated (baseline) PDEs to a varying degree (Fig. 1a, b). In treated explant tissues, ER:PR-B interactions were only detected in the presence of both hormones $\left(E_{2}+\right.$ R5020) (Fig. 1a, b). There was no detectable difference in levels of ER and PR among explants by immunohistochemistry in the four treatment groups for any case. In T47D cells, ER and PR have been previously shown to interact in the presence of $E_{2}$ and progestogen using coimmunoprecipitation technologies. ${ }^{10-12}$ To show this in situ, T47D cells were treated for $24 \mathrm{~h}$ with vehicle, $E_{2}$, progesterone or the combination of $E_{2}$ and progesterone under steroid-depleted conditions. As observed in the treated PDEs, ER:PR-B interactions were only detected in FFPE cell pellets of T47D cells treated with the combination of E2 and progesterone (Fig. 1c). Again, there was no detectable change in ER and PR by immunohistochemistry among treatments. Collectively these data show that ER:PR-B interactions only occur in PDEs when both receptors are acutely ligand-activated.

Association between ER:PR-B interactions, PR-B expression by PLA and ER and PR immunohistochemistry in breast cancers

The ER:PR-B PLA was applied to 229 primary tumours arranged in quadruplicate on a TMA and scored by counting the number of signals per tumour cell nucleus. The vast majority of interactions were intranuclear and very occasional cases demonstrated cytoplasmic interactions. All cases had detectable ER by immunohistochemistry at a clinically relevant level ( $>1 \%$ positive tumour nuclei). ${ }^{34}$ There was a significant positive correlation between ER: PR-B interactions and PR expression $(p=0.003)$ (Table 1). Similarly, ER:PR-B interactions and ER expression were positively correlated $(p=0.001$ ) (Table 1). In 44 tumours that were negative for PR by immunohistochemistry (Allred score $0-2$; representing weak positive staining in less than $1 \%$ of tumour cells), the median number of ER:PR-B interactions was 2.28 (inter-quartile range 1.8-10.3), significantly lower than the number of interactions in cases with a PR Allred score of 3 or more (median 6.45 signals per cell, inter-quartile range $0.26-7.99, p=0.001$ ) (Fig. 2b). However, the number of ER:PR-B interactions detected was not absolutely dependent on relative expression of PR by IHC; many cases with high levels of PR expression showed very few detectable ER:PR-B interactions, and conversely there were cases with significant numbers of ER:PR-B interactions in the absence of detectable PR expression by IHC (Fig. 2a).

We postulated that detection of ER:PR-B interactions in the absence of PR immunostaining was due to the increased sensitivity of the PLA over the immunohistochemical assay for PR. To investigate this, we developed a PLA to determine specific expression of the PR-B isoform (Fig. 2a). PR-B expression was highly correlated with PR (A/B) expression by IHC $(r=0.807 ; p=<$ 0.001 ) (Fig. 2C). There was a significant correlation between PR-B expression and ER:PR-B interactions $(r=0.352 ; p=<0.001)$ (Fig. 2d). In the 44 patients that were negative for PR by IHC, there was significant positive correlation between ER:PR-B interactions and PR-B expression by PLA $(r=0.608 ; p=<0.001)$ (Fig. 2e), demonstrating that detection of PR-B by PLA is more sensitive than detecting PR by IHC. 
a
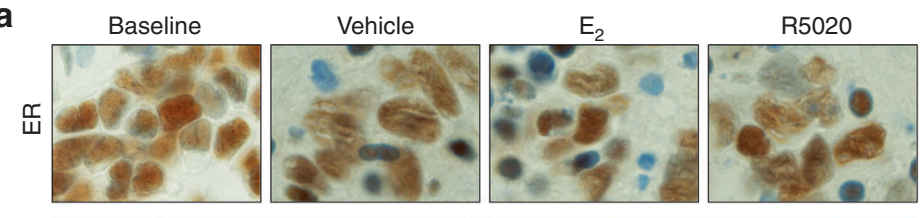

$\mathrm{E}_{2}+\mathrm{R} 5020$

$\stackrel{\Upsilon 1}{0}$
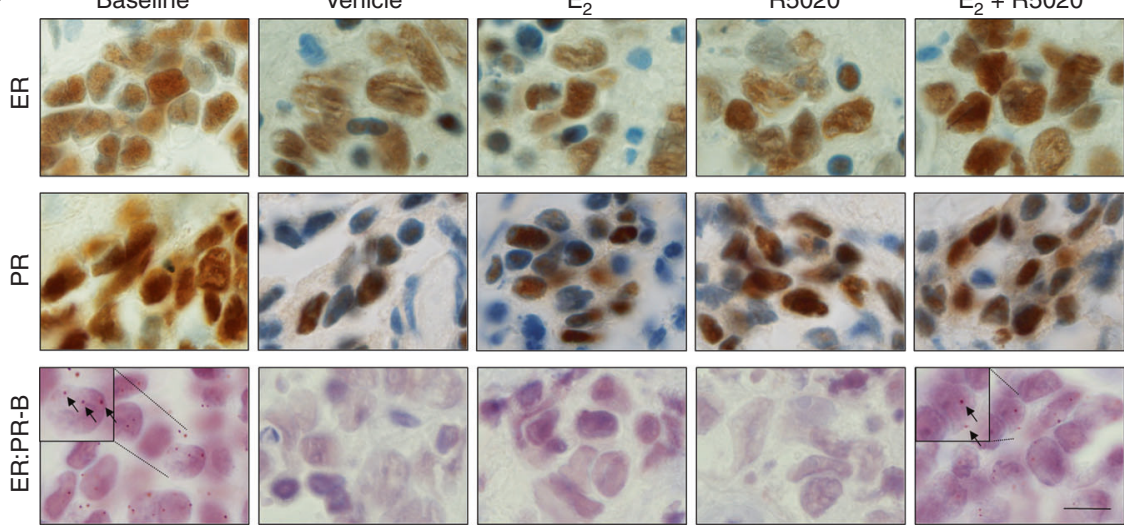

b

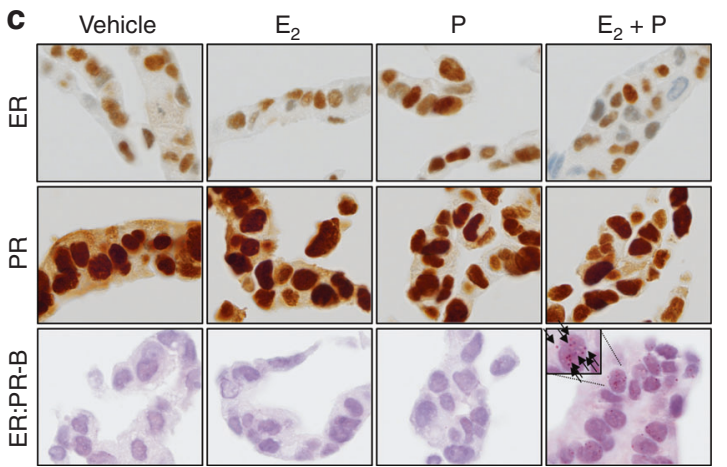

Fig. 1 Development of a proximity ligation assay to detect interactions between the oestrogen receptor- $\alpha$ and progesterone receptor-B (ER: PR-B) in patient derived breast cancer explants and T47D cells. a Breast cancer explants were treated with either Vehicle, oestradiol ( $\left.E_{2}\right)$, R5020 or the combination of $E_{2}$ and R5020. Explants were immunohistochemically stained for the oestrogen receptor- $\alpha$ (ER), progesterone receptor (PR) and the ER:PR-B proximity ligation assay (PLA). Scale bar (bottom right, applies to all images) is $10 \mu \mathrm{M}$. $\mathbf{b}$ Bar graph indicates mean number of ER:PR-B interactions and standard deviation per cell for three explants with each condition. $c$ T47D cells were treated with either Vehicle, $E_{2}$, progesterone $(P)$ or the combination of $E_{2}$ and $P$. Cells were immunohistochemically stained for ER, PR and stained using the ER:PR-B PLA

Table 1. Correlations between ER:PR-B interactions (signals/cell) and clinicopathological variables

\begin{tabular}{llll}
\hline Variable & Spearman's $r$ & $p$ & $N$ \\
\hline Age & 0.229 & 0.001 & 227 \\
Post-menopausal & 0.133 & 0.045 & 227 \\
T-stage & 0.056 & 0.405 & 227 \\
N-stage & -0.077 & 0.247 & 227 \\
Grade & -0.144 & 0.030 & 227 \\
Mitotic score & -0.198 & 0.003 & 227 \\
Multiple tumours & -0.039 & 0.555 & 227 \\
Mastectomy & -0.056 & 0.402 & 227 \\
Axillary clearance & -0.020 & 0.769 & 227 \\
Adjuvant chemotherapy & -0.058 & 0.382 & 226 \\
Adjuvant radiation & 0.019 & 0.778 & 224 \\
Adjuvant endocrine therapy & -0.044 & 0.511 & 226 \\
ER expression (allred score) & 0.567 & 0.001 & 227 \\
PR expression (allred score) & 0.199 & 0.003 & 227 \\
PR-B expression (PLA signals) & 0.352 & 0.001 & 227 \\
\hline The $p$ value quoted is the result of a & 2 -tailed Spearman's correlation. $N$ is \\
the number of patients with pairwise non-missing values & & \\
\hline
\end{tabular}

Correlations between ER:PR-B interactions and clinical and pathological variables

There was a positive correlation between ER:PR-B interactions and age $(p=0.001)$, with a higher number of interactions in postmenopausal women (Table 1). Higher ER:PR-B interactions were correlated with lower tumour grade $(p=0.030)$ and lower mitotic score $(p=0.003)$. There was no significant correlation with T-stage, $\mathrm{N}$-stage, the presence of multiple tumours, or type of surgical or adjuvant treatment.

Association of ER and PR immunohistochemistry and ER:PR-B interactions with relapse-free survival

In the cohort as a whole, absent PR immunohistochemistry was associated with poorer relapse free survival (log-rank $p=0.021$ ) (Fig. 3a). ROC curve analysis was used to determine an optimal cut-off for the number of ER:PR-B interactions per cell in patients who had received adjuvant tamoxifen or an $\mathrm{Al}$ using relapse status as the dependent variable (supplementary material, Figure S1). In Al-treated patients, the area under the curve was $0.701(p=0.0013)$ with a cut-off of 5 signals per cell. The sensitivity for detecting relapse was $76.9 \%$ and specificity was 63.4\%. Similar ROC curve analysis for ER expression (Allred Score) showed no significant association with relapse (supplementary material, Figure S2). When the whole cohort of patients was dichotomised into low $(\leq 5)$ and high ER:PR-B interactions, 

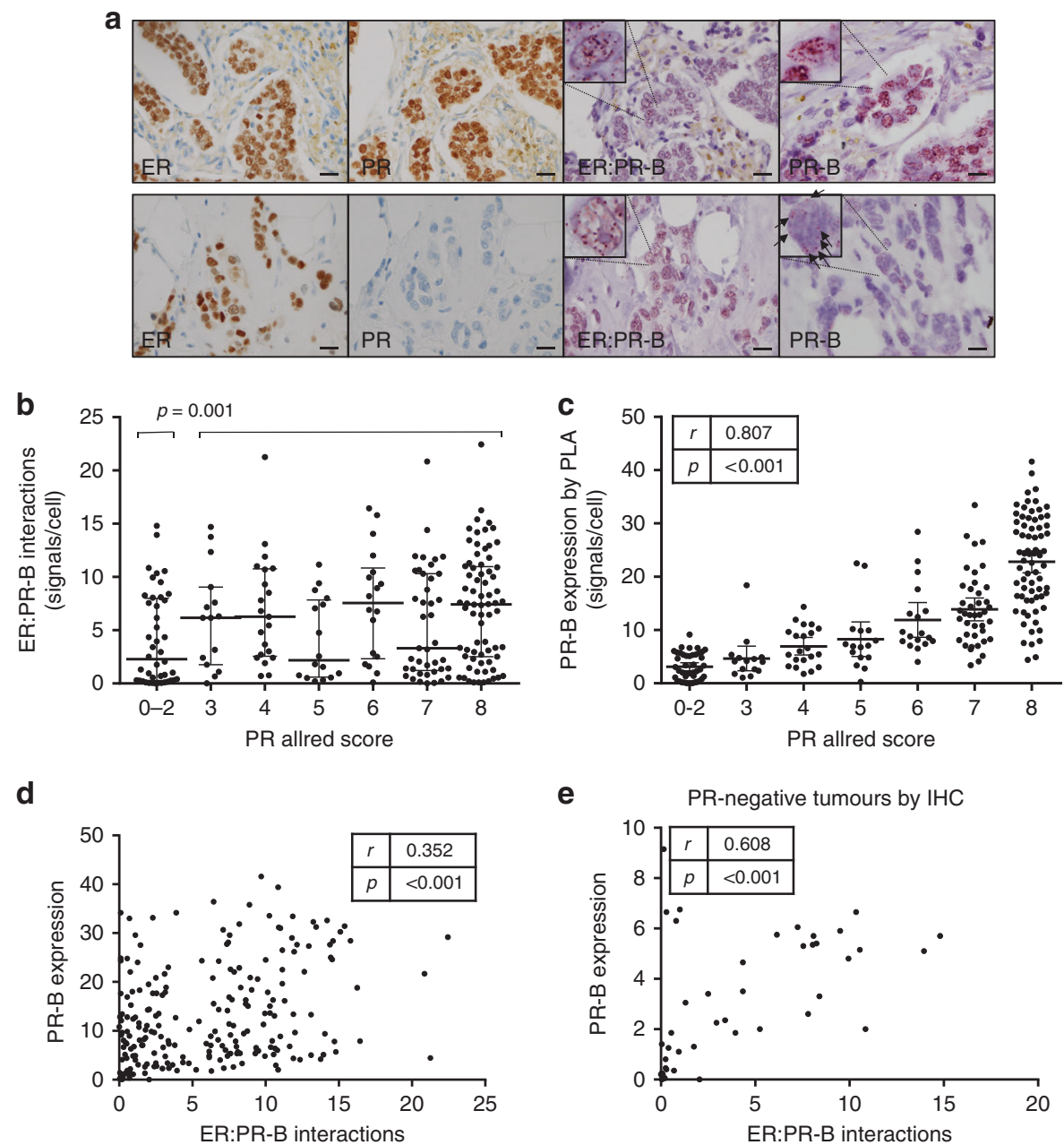

Fig. 2 Associations between total PR expression, ER:PR-B interactions and PR-B expression by proximity ligation assay and example photomicrographs. 227 patients with ER+, HER2- breast cancer with paired evaluable expression of PR by immunohistochemistry (Allred score), ER:PR-B interactions and PR-B expression by proximity ligation assay (signals/cell) are represented. a Example immunostains for ER, PR and PLA images for two patients. Scale bar is $20 \mu \mathrm{M}$. b Association between ER:PR-B interactions and PR expression (A/B) by immunohistochemistry. Line indicates median with interquartile range. $P$ value is the result of the Mann-Whitney $U$ test. $c$ Association between PR-B expression by PLA and PR expression (A/B) by immunohistochemistry. d Association between PR-B expression by PLA and ER: PR-B interactions. e Association between PR-B expression by PLA and ER:PR-B interactions in 44 patients negative for PR (A/B) by immunohistochemistry

those with low ER:PR-B interactions had significantly poorer relapse free survival (log-rank $p=0.003$ ) (Fig. 3a).

Univariate and multivariate Cox regression analyses of variables affecting relapse

In an analysis of the whole cohort of patients, higher pathological $\mathrm{T}$-stage and $\mathrm{N}$-stage were significantly associated with relapse in univariate analysis (Table 2). Patients that underwent chemotherapy and took prescribed endocrine therapy had a significantly reduced risk of relapse. Absent PR expression was significantly associated with relapse (HR 2.028, Cl 1.100-3.731, $p=0.024)$ and low levels of ER:PR-B interactions had a higher risk of relapse (HR $2.463, \mathrm{Cl} 1.333-4.545, p=0.004)$. There was no significant prognostic effect of age, grade, mitotic score, histologic type, multiple tumours, type of surgery, adjuvant radiotherapy or class of endocrine agent taken.

ROC analysis was used to determine an optimal cut-off for the number of PR-B signals per cell in patients who had received adjuvant tamoxifen or an Al using relapse status as the dependent variable (supplementary material, Figure S3). Using dichotomised expression with a cut-off of 13.5 signals per cell, sensitivity for detecting relapse was $57.1 \%$ and specificity was $92.9 \%$ for tamoxifen treated patients and $43.1 \%$ and $84.6 \%$ respectively for Al-treated patients. Low PR-B expression was significantly associated with relapse (HR 3.636, $\mathrm{Cl} 1.543-8.621, p=0.003$ ).

In a multivariate model that included dichomised levels of ER: PR-B interactions (low $\leq 5$; high $>5$ ) and other standard clinical factors significant in univariate analysis, only low levels of ER:PR-B interaction ( $\mathrm{HR} 2.475, \mathrm{Cl} 1.297-4.717, p=0.006)$, higher T-stage (HR 3.031, Cl 1.063-8.649, $p=0.038$ ) and endocrine therapy (HR $0.335, \mathrm{Cl} 0.121-0.926, p=0.035)$ were independent prognostic factors associated with relapse (second column, Table 2). Absent $\mathrm{PR}$ expression, $\mathrm{N}$-stage and having adjuvant chemotherapy were not significant independent prognostic factors for relapse. In a multivariate model that included both ER:PR-B interactions and PR-B expression, both ER:PR-B interactions and PR-B expression were independent prognostic factors associated with relapse (third column, Table 2).

Prognostic effect of ER:PR-B interactions and PR-B expression stratified by type of adjuvant endocrine agent

In an exploratory analysis of ER:PR-B interactions stratified by type of endocrine agent, a low frequency of ER:PR-B interaction was associated with relapse in patients taking Als as adjuvant therapy 
a

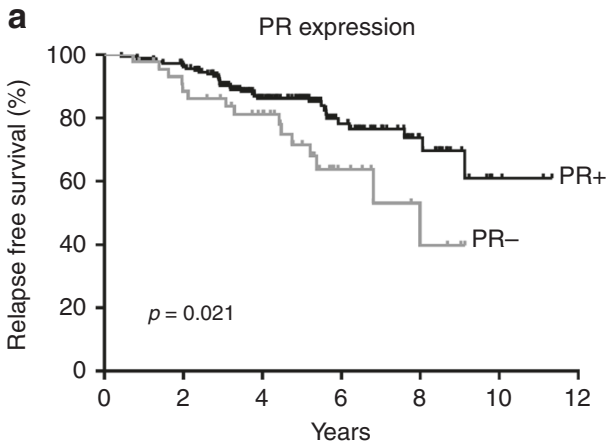

Number at risk

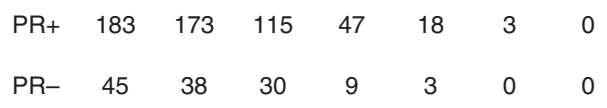

b

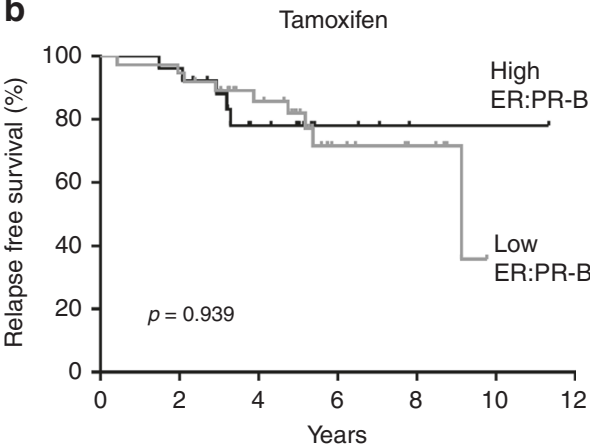

Number at risk

$\begin{array}{lllllll}\begin{array}{l}\text { High } \\ \text { ER-PR }\end{array} & 26 & 13 & 4 & 1 & 0 & 0 \\ \text { Low }_{\text {ER-PR }}^{37} & 35 & 25 & 9 & 5 & 0 & 0\end{array}$

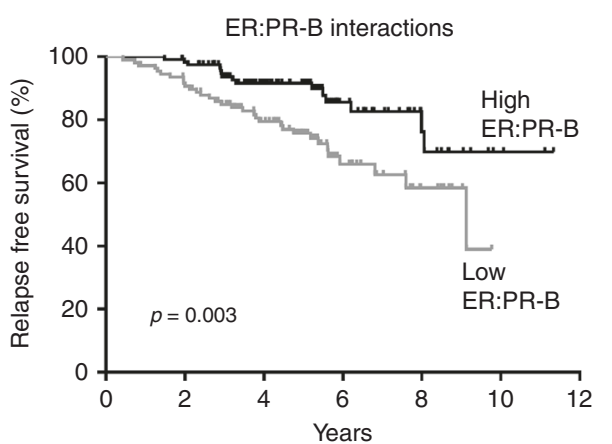

Number at risk
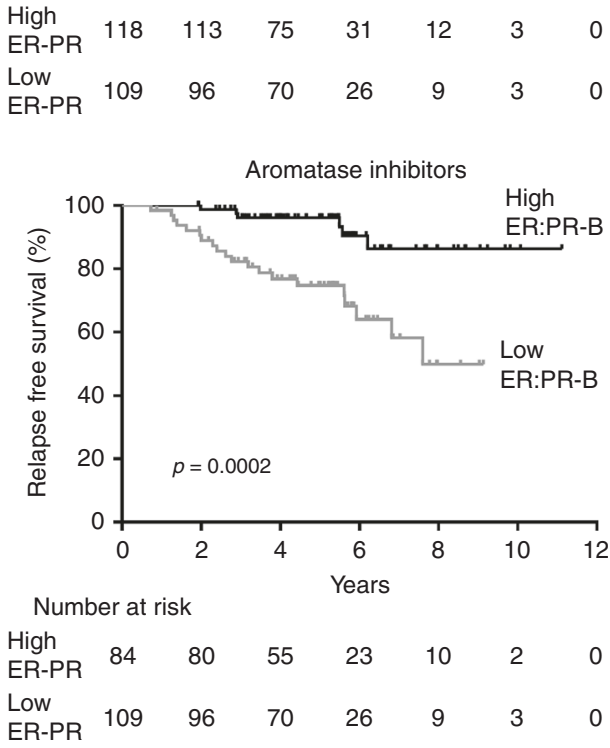

Fig. 3 Kaplan-Meier curves by PR expression and ER:PR-B interactions (a) and ER:PR-B interactions stratified by type of adjuvant endocrine agent taken (b). $P$ values quoted are the result of the log-rank test

(log-rank $p=0.0002$ ), but not with those taking tamoxifen (logrank $p=0.939)$ (Fig. 3b). This equated to a hazard ratio of $4.831(\mathrm{Cl}$ $1.942-12.048, p=0.001$ ) for patients with low ER:PR-B interactions taking an Al (Table 3). A test for interaction was significant ( $p=$ 0.031 ). Patients taking adjuvant endocrine therapy had significant clinical and pathological differences depending on the type of agent (supplementary material, Table S2). Patients on tamoxifen were younger, more likely to be pre-menopausal, had lower Tstage and a higher proportion had tumours with the histology 'no special type'. In patients taking adjuvant tamoxifen, there was a trend towards a greater proportion of tumours with low levels of ER:PR-B interactions $(p=0.051)$.

When levels of PR-B were stratified by type of endocrine agent taken, low PR-B expression was only significantly associated with relapse in women taking adjuvant tamoxifen (HR $8.929, \mathrm{Cl}$ 1.164-66.667, $p=0.035$ ) (Table 3). A test for interaction was not significant $(p=0.355)$ There was no significant association between PR expression stratified by type of adjuvant endocrine agent and relapse in patients either taking an Al or tamoxifen (supplementary material, Table S3).

\section{DISCUSSION}

Herein we report the development and application of a novel ER: PR-B interaction assay using PLA that can be used in FFPE breast cancer tissue sections and show that the frequency of these interactions is able to predict response to adjuvant Al therapy. In breast cancer cells in vitro and in treated PDEs, the interaction between ER and PR-B was dependent on ligand-activation of both ER and PR-B by E2 and progestogen, respectively. No interactions were detected in the presence of a single agonist ligand. This finding is consistent with ligand activation of both steroid receptors being required to promote the formation of a functional ER:PR-B complex. ${ }^{12,13}$ In multivariate analysis, we found that PR expression determined by an accredited diagnostic laboratory was not an independent predictive factor for relapse following adjuvant Al therapy, consistent with previous studies. ${ }^{6-8}$ However, low levels of ER:PR-B interactions were predictive of relapse in the Al setting. These findings suggest that ER-PR interactions are a major determinant of the prognostic value of PR expression.

PR-B expression determined by PLA strongly correlated with the levels of total PR by immunohistochemistry, consistent with previous reports. ${ }^{18,20}$ In the 44 cases negative for PR by immunohistochemistry, there was a correlation between PR-B expression determined by PLA and ER:PR-B interactions suggesting the presence of low levels of PR-B in the tumours was sufficient to interact with ER. Lower levels of PR-B were associated with a significantly increased risk of relapse, which in sub-group analysis was limited to patients on adjuvant tamoxifen. This is consistent with previous reports showing lower PR-A to PR-B ratios were associated with poorer survival on tamoxifen. ${ }^{18,39}$ In the cohort analysed as a whole, multivariate analysis demonstrated PR-B expression and ER:PR-B interactions were both independent predictors of relapse, and in a sub-group analysis they differentially predict risk dependent on type of adjuvant therapy. ER:PR-B interactions were not associated with relapse in tamoxifen treated 
Table 2. Univariate and multivariate Cox regression analysis of clinicopathological factors influencing relapse-free survival in ER+, HER2-, node positive breast cancer patients

\begin{tabular}{|c|c|c|c|c|c|c|c|c|c|}
\hline \multirow[t]{2}{*}{ Variable } & \multicolumn{3}{|c|}{ Univariate analysis } & \multicolumn{3}{|c|}{$\begin{array}{l}\text { Multivariate analysis including } \\
\text { ER:PR-B interactions }\end{array}$} & \multicolumn{3}{|c|}{$\begin{array}{l}\text { Multivariate analysis including } \\
\text { ER:PR-B interactions and PR-B } \\
\text { expression }\end{array}$} \\
\hline & $\mathrm{HR}$ & $95 \% \mathrm{Cl}$ & $p$ Value & $\mathrm{HR}$ & $95 \% \mathrm{Cl}$ & $p$ Value & $\mathrm{HR}$ & $95 \% \mathrm{Cl}$ & $p$ Value \\
\hline Age & 1.002 & $0.979-1.026$ & 0.835 & & & & & & \\
\hline T-stage $\mathrm{pT} 2 / 3 / 4$ vs $\mathrm{pT} 1$ & 3.383 & $1.214-9.427$ & 0.020 & 3.031 & $1.063-8.649$ & 0.038 & 3.209 & $1.128-9.130$ & 0.029 \\
\hline $\mathrm{N}$-stage $\mathrm{pN} 2 / 3$ vs $\mathrm{pN} 1$ & 1.999 & $1.122-3.561$ & 0.019 & 1.623 & $0.890-2.961$ & 0.114 & 1.536 & $0.842-2.802$ & 0.162 \\
\hline Grade 3 vs 1 & 1.804 & $0.862-3.774$ & 0.117 & & & & & & \\
\hline Mitotic score 2 vs 1 & 1.139 & $0.574-2.261$ & 0.710 & & & & & & \\
\hline Mitotic score 3 vs 1 & 1.175 & $0.801-1.724$ & 0.408 & & & & & & \\
\hline Histology Lobular vs NST & 1.448 & $0.678-3.091$ & 0.339 & & & & & & \\
\hline Histology Other vs NST & 1.433 & $0.722-2.841$ & 0.304 & & & & & & \\
\hline Adjuvant radiation vs not & 0.740 & 0.3501 .564 & 0.431 & & & & & & \\
\hline Adjuvant endocrine therapy vs not & 0.305 & $0.129-0.720$ & 0.007 & 0.299 & $0.116-0.767$ & 0.012 & 0.374 & $0.146-0.954$ & 0.040 \\
\hline Aromatase inhibitor vs tamoxifen & 0.803 & 0.3861 .669 & 0.557 & & & & & & \\
\hline PR expression Negative vs positive & 2.028 & $1.100-3.731$ & 0.024 & 1.543 & $0.789-3.021$ & 0.205 & 1.220 & $0.619-2.404$ & 0.566 \\
\hline PR-B expression Low vs high & 3.636 & $1.543-8.621$ & 0.003 & & & & 2.841 & $1.134-7.143$ & 0.026 \\
\hline ER:PR-B interactions low vs high & 2.463 & $1.333-4.545$ & 0.004 & 2.475 & $1.297-4.717$ & 0.006 & 2.101 & $1.092-4.049$ & 0.026 \\
\hline
\end{tabular}

patients. This may be due to the small numbers of tamoxifentreated patients in this cohort or the mechanism of action of tamoxifen. Tamoxifen directly binds ER and promotes interactions with corepressors, ${ }^{40}$ possibly disrupting interactions with PR-B.

A higher frequency of ER:PR-B interactions was detected in post-menopausal women. Whilst circulating levels of oestrogen and progesterone both dramatically decline with the onset of menopause, ${ }^{24}$ peripheral conversion of circulating androgens to oestrogen increases in many tissues, including the breast. ${ }^{41,42}$ Indeed, tissue levels of oestrogen in the post-menopausal breast are sufficient to promote the development of ER+ breast cancer, which forms the basis of clinical benefit from Als. ${ }^{43}$ However, peripheral production of progestogens within post-menopausal tissues is not well characterised. Progesterone is detectable in the breast tissue of post-menopausal women with breast cancer using highly sensitive mass-spectrometry methodology and has been recently reported to represent $2.1 \%$ of total steroids extracted, about twice the percentage represented by oestrogen $(1 \%)^{41}$ Since there is no known mechanism of local production of progesterone in breast tissue, the major difference in ER:PR-B interactions between postmenopausal tumours in our study is most likely due to differences in the circulating levels of progesterone and the level of progesterone metabolising enzymes expressed by the tumour or cells in the microenvironment. In both normal and malignant breast tissues, progesterone can be metabolised into 5a-pregnanes and 4-pregnenes by $5 a-$ reductase and 3a-hydroxysteroidoxidoreductase enzymes. ${ }^{44}$ Interestingly, we observed some tumours with detectable ER by immunohistochemistry and PR-B by PLA that showed no evidence of interaction by PLA. This finding likely reflects the degree of interaction between ER and PR-B being more dependent on the availability of agonist ligands than the receptor levels in the individual tumours. Ligand-activated PR-B promotes interaction with ER to reprogram the ER-associated cistrome and induce a transcriptome associated with good clinical outcome ${ }^{12}$; herein we demonstrate that this interaction exists in clinical tissues and is associated with increased relapse-free survival. These findings support the concept currently being tested in clinical trials that promoting ER:PR-B interactions by therapeutic administration of a progestogen may be an effective adjuvant treatment strategy for ER+ breast cancer. ${ }^{28,45,46}$ Indeed, assessment of ER:PR-B interactions using our new assay may be a means of monitoring treatment response in those trials.

Low levels of ER:PR-B interactions were observed in tumours with more aggressive features (higher tumour grade and increased numbers of mitotic figures). This is consistent with ER: PR-B interactions being associated with PR-B reprogramming of ER chromatin binding to promote a transcriptional output associated with tumour-suppressive processes including differentiation and cell death. ${ }^{12-14}$ One variable affecting ER:PR-B interactions in breast tumours is that the PR gene is often lost in ER+ tumours due to deletion ${ }^{12,47-49}$ or its expression is reduced due to hypermethylation of the PGR gene locus. ${ }^{50}$ In particular, PR expression typically is lost or reduced in more aggressive luminal $B$ breast cancers, ${ }^{12,13}$ which like all ER+ cancers are more common in postmenopausal women. ${ }^{16}$ In this situation, PR-B reprograming of ER signalling would not occur, leading to maintenance of a growth stimulatory state and a poor disease outcome. In our study we do not know the status of the PR gene in tumours that did not have detectable PR by immunohistochemistry. However, we found that some of these tumours had detectable ER:PR-B interactions and PR-B expression by PLA, indicating an intact PR gene. In current trials involving progestogen therapy, tumours that lack PR by IHC would be deemed ineligible for treatment. Use of our PR-B and ER:PR-B PLA could represent a more sensitive assay to determine eligibility criteria for such trials. 
Table 3. Cox regression analysis of ER:PR-B interactions and PR-B expression influencing relapse-free survival stratified by adjuvant endocrine agent class taken

\begin{tabular}{|c|c|c|c|c|c|}
\hline Endocrine agent & $\begin{array}{l}\text { ER:PR-B } \\
\text { interaction }\end{array}$ & $\begin{array}{l}\text { Relapse } \\
\text { (\%) }\end{array}$ & $\mathrm{HR}$ & $95 \% \mathrm{Cl}$ & $p$ Value \\
\hline \multirow[t]{2}{*}{ Tamoxifen } & Low & $9(24.3)$ & 1.043 & $0.348-3.135$ & 0.939 \\
\hline & High & $5(19.2)$ & & & \\
\hline \multirow{2}{*}{$\begin{array}{l}\text { Aromatase } \\
\text { inhibitor }\end{array}$} & Low & $20(30.8)$ & 4.831 & $1.942-12.048$ & 0.001 \\
\hline & High & $6(7.1)$ & & & \\
\hline \multirow{2}{*}{$\begin{array}{l}\text { Test for } \\
\text { interaction }\end{array}$} & & & & & 0.031 \\
\hline & $\begin{array}{l}\text { PR-B } \\
\text { expression }\end{array}$ & $\begin{array}{l}\text { Relapse } \\
\text { (\%) }\end{array}$ & HR & $95 \% \mathrm{Cl}$ & $p$ Value \\
\hline \multirow[t]{2}{*}{ Tamoxifen } & Low & $13(36.1)$ & 8.929 & $1.164-66.667$ & 0.035 \\
\hline & High & $1(3.7)$ & & & \\
\hline \multirow{2}{*}{$\begin{array}{l}\text { Aromatase } \\
\text { inhibitor }\end{array}$} & Low & $22(23.9)$ & 2.874 & $0.984-8.403$ & 0.053 \\
\hline & High & $4(7.0)$ & & & \\
\hline $\begin{array}{l}\text { Test for } \\
\text { interaction }\end{array}$ & & & & & 0.355 \\
\hline
\end{tabular}

Percentages refer to the number of patients with high or low ER:PR-B interactions or PR-B expression that had relapsed on endocrine treatment. Bold indicates significant $p$ values

Patients with PR-negative tumours consistently have a poorer prognosis than those with $\mathrm{PR}+$ tumours, ${ }^{51}$ regardless of adjuvant endocrine agent taken. ${ }^{6-8}$ We also found that patients with tumours that were PR-negative by immunohistochemistry had a poorer prognosis, but the level of ER:PR-B interactions was more prognostic for relapse. ER levels were not associated with relapse. The outcomes of patients in our cohort, which only included node-positive patients with ER+ breast cancer, are highly dependent on the efficacy of systemic adjuvant treatments, including chemotherapy and endocrine therapy. Non-compliance with endocrine therapy is well-recognised as being associated with adverse disease outcomes. ${ }^{52}$ Effective adjuvant endocrine therapy is clearly critical in preventing relapse in node-positive ER + breast cancer.

When stratified by type of endocrine agent taken, the prognostic effect of ER:PR-B interactions was limited to patients on Als, predominantly in post-menopausal women. The low numbers of patients in the tamoxifen treated group, most of whom were pre-menopausal, may be partially responsible for the lack of prognostic significance of ER:PR-B interactions. A significant test for interaction indicated that ER:PR-B interactions may be predictive of Al efficacy. These findings need to be replicated in prospective randomised controlled trials to determine whether ER:PR-B interactions or PR-B expression by PLA may be used to select for adjuvant endocrine treatment. There are several large phase III trials of cyclin-dependent kinase (CDK) $4 / 6$ inhibitors in combination with endocrine therapy for ER-positive $(E R+)$ early stage breast cancer. ${ }^{53-55}$ ER:PR-B interactions may also serve as predictors of benefit to CDK4/6 inhibitors by identifying patients likely to relapse on standard adjuvant endocrine therapy.

In conclusion, while there is abundant clinical data showing that PR agonists are beneficial in postmenopausal patients with advanced $\mathrm{ER}+$ breast cancer, ${ }^{56-59}$ their use as an adjuvant therapy is not established and trials are in progress. ${ }^{28}$ Several recent preclinical studies provide compelling evidence that the key to effective progestogen therapy is the ability of activated PR (specifically PR-B) to reprogram the genomic activity of activated ER. Herein, we describePLA assays which can detect the interaction of ER and PR-B and expression of PR-B in FFPE tissues that could feasibly be automated to facilitate use in diagnostic histopathology laboratories. We further show that assessment of these interactions could have clinical value and propose that measuring the level of ER:PR-B interactions may predict benefit from progestogen treatment and aid patient selection in future randomised clinical trials of progestogens. Quantifying PR-B levels by PLA appears to be a refinement on PR IHC, which may explain why it is more prognostic for relapse in patients on adjuvant tamoxifen. Finally, we find that ER:PR-B interactions are associated with relapse in patients taking adjuvant Als, suggesting that ER:PR$B$ interactions may have utility in predicting efficacy of Als as well as response to progestogen therapy.

\section{ACKNOWLEDGEMENTS}

The authors thank the following people for their contributions to this study: Yanlin Liu for assembly of the database used to collate patient information. Lauren Furnas for help with block retrieval. Brenton Seidl for help with retrieving patient information. Geraldine Lavin-Law for technical work involving the patient-derived explants. This work was supported by grants from the Royal College of Pathologists of Australasia Foundation (CS), National Health and Medical Research Council of Australia (NHMRC) (ID 1130077 WDT and TEH), and Cancer Australia/National Breast Cancer Foundation (ID 1043497; WDT, TEH). CS is supported by a Betty McGrath Fellowship from Mater Foundation. $\mathrm{CL}$ is a recipient of $\mathrm{PhD}$ scholarships from the Australian Government (Australian Postgraduate Award) and Royal College of Pathologists of Australasia (RCPA Foundation Postgraduate Research Fellowship). WDT is also supported by a grant from the National Breast Cancer Foundation (PS-15041). TEH is supported by a Career Development Fellowship from the Royal Adelaide Hospital Research Foundation (Adelaide, Australia). The project also has received funding from the Mater Foundation (Queensland, Australia) and The Hospital Research Foundation (Adelaide, Australia). The Translational Research Institute is supported by a grant from the Australian Government.

\section{AUTHOR CONTRIBUTIONS}

C.E.S, J.A, T.E.H. and W.D.T. conceived the study. C.E.S, M.G. and T.E.H. performed the experiments. C.E.S. and C.L. scored the slides. C.E.S. and M.G. performed statistical analyses. Patient data was collected by K.M, C.P, C.S. and N.W. C.E.S. wrote the paper with major editing from W.D.T, T.E.H. and J.E.A. and minor editing and final approval of the submitted version by all authors.

\section{ADDITIONAL INFORMATION}

Supplementary Information is available for this paper at https://doi.org/10.1038/ s41416-018-0331-3.

Competing interests: KM reports receiving travel expenses from Roche. CS reports receiving compensation for being on advisory boards for Roche and AstraZeneca. NW reports stock ownership (CSL), receiving travel/expenses from Roche and research funding from Medivation. The remaining authors declare that they have no conflict of interest.

Ethics approval and consent to participate: for patient-derived explant studies, tumour samples were obtained following informed consent from women undergoing surgery for breast cancer at the Burnside War Memorial Hospital, Adelaide. This study was approved by the University of Adelaide Human Research Ethics Committee (approval numbers: $\mathrm{H}-065-2005 ; \mathrm{H}-169-2011)$. For the retrospective cohort, the use of clinical information and tumour blocks was approved by the Mater Health Services Human Research Ethics Committee with a waiver of consent (approval number: HREC/ $15 / \mathrm{MHS} / 123)$. The study was performed in accordance with the Declaration of Helsinki.

Availability of data and materials: To protect patient privacy, all patient data have been de-identified and reported in aggregate. De-identified patient data are available from the authors on request by e-mail.

Note: This work is published under the standard license to publish agreement. After 12 months the work will become freely available and the license terms will switch to a Creative Commons Attribution 4.0 International (CC BY 4.0).

\section{REFERENCES}

1. Kohler, B. A. et al. Annual report to the nation on the status of cancer, 1975-2011, featuring incidence of breast cancer subtypes by race/ethnicity, poverty, and state. J. Natl Cancer Inst. 107, djv048 (2015). E-pub ahead of print 2015/04/01. 
2. Howlader, N., Cronin, K. A., Kurian, A. W. \& Andridge, R. Differences in breast cancer survival by molecular subtypes in the United States. Cancer Epidemiol. Biomark. \& Prev. a Publ. Am. Assoc. Cancer Res. cosponsored Am. Soc. Prev. Oncol. 27, 619-626 (2018). E-pub ahead of print 2018/03/30.

3. Horwitz, K. B. \& McGuire, W. L. Estrogen control of progesterone receptor in human breast cancer. Correlation with nuclear processing of estrogen receptor. J. Biol. Chem. 253, 2223-2228 (1978). E-pub ahead of print 1978/04/10.

4. Snell C. E., et al. Absent progesterone receptor expression in the lymph node metastases of ER-positive, HER2-negative breast cancer is associated with relapse on tamoxifen. J. Clin. Pathol. 70, 954-960 (2017). E-pub ahead of print 2017/04/19.

5. Stendahl, M. et al. High progesterone receptor expression correlates to the effect of adjuvant tamoxifen in premenopausal breast cancer patients. Clin. Cancer Res. Off. J. Am. Assoc. Cancer Res. 12, 4614-4618 (2006). E-pub ahead of print 2006/08/11.

6. Dowsett, M. et al. Relationship between quantitative estrogen and progesterone receptor expression and human epidermal growth factor receptor 2 (HER-2) status with recurrence in the arimidex, tamoxifen, alone or in combination trial. $J$. Clin. Oncol. Off. J. Am. Soc. Clin. Oncol. 26, 1059-1065 (2008). E-pub ahead of print 2008/01/30.

7. Thurlimann, B. et al. A comparison of letrozole and tamoxifen in postmenopausal women with early breast cancer. N. Engl. J. Med. 353, 2747-2757 (2005). E-pub ahead of print 2005/12/31.

8. Viale, G. et al. Prognostic and predictive value of centrally reviewed expression of estrogen and progesterone receptors in a randomized trial comparing letrozole and tamoxifen adjuvant therapy for postmenopausal early breast cancer: BIG 198. J. Clin. Oncol. Off. J. Am. Soc. Clin. Oncol. 25, 3846-3852 (2007). E-pub ahead of print 2007/08/08.

9. Early Breast Cancer Trialists' Collaborative Group (EBCTCG) Aromatase inhibitors versus tamoxifen in early breast cancer: patient-level meta-analysis of the randomised trials. Lancet (London, England) 2015; 386: 1341-1352; e-pub ahead of print 2015/07/28.

10. Ballare, C. et al. Two domains of the progesterone receptor interact with the estrogen receptor and are required for progesterone activation of the c-Src/Erk pathway in mammalian cells. Mol. Cell. Biol. 23, 1994-2008 (2003). E-pub ahead of print 2003/03/04.

11. Daniel, A. R. et al. Progesterone receptor-B enhances estrogen responsiveness of breast cancer cells via scaffolding PELP1- and estrogen receptor-containing transcription complexes. Oncogene 34, 506-515 (2015). E-pub ahead of print 2014/01/29.

12. Mohammed, $\mathrm{H}$. et al. Progesterone receptor modulates ERalpha action in breast cancer. Nature 523, 313-317 (2015). E-pub ahead of print 2015/07/15

13. Singhal, H. et al. Genomic agonism and phenotypic antagonism between estrogen and progesterone receptors in breast cancer. Sci. Adv. 2, e1501924 (2016). E-pub ahead of print 2016/07/08.

14. Finlay-Schultz, J. et al. Breast cancer suppression by progesterone receptors is mediated by their modulation of estrogen receptors and RNA polymerase III. Cancer Res. 77, 4934-4946 (2017). E-pub ahead of print 2017/07/22.

15. Giangrande, P. H. \& McDonnell, D. P. The A and B isoforms of the human progesterone receptor: two functionally different transcription factors encoded by a single gene. Recent Prog. Horm. Res. 54, 291-313 (1999). Discussion 313-294; epub ahead of print 1999/11/05

16. Voduc, K. D. et al. Breast cancer subtypes and the risk of local and regional relapse. J. Clin. Oncol. Off. J. Am. Soc. Clin. Oncol. 28, 1684-1691 (2010). E-pub ahead of print 2010/03/03.

17. Graham, J. D. et al. Characterization of progesterone receptor A and B expression in human breast cancer. Cancer Res. 55, 5063-5068 (1995). E-pub ahead of print 1995/11/01.

18. Hopp, T. A. et al. Breast cancer patients with progesterone receptor PR-A-rich tumors have poorer disease-free survival rates. Clin. Cancer Res. Off. J. Am. Assoc. Cancer Res. 10, 2751-2760 (2004). E-pub ahead of print 2004/04/23.

19. Rojas P. A., et al. Progesterone receptor isoform ratio: a breast cancer prognostic and predictive factor for antiprogestin responsiveness. J. Natl Cancer Inst. 7, djw317, 2017. E-pub ahead of print 2017/04/05.

20. Bamberger, A. M., Milde-Langosch, K., Schulte, H. M. \& Loning, T. Progesterone receptor isoforms, PR-B and PR-A, in breast cancer: correlations with clinicopathologic tumor parameters and expression of AP-1 factors. Horm. Res. 54, 32-37 (2000). E-pub ahead of print 2001/02/22.

21. Singhal, H. et al. Progesterone receptor isoforms, agonists and antagonists differentially reprogram estrogen signaling. Oncotarget 9, 4282-4300 (2018). E-pub ahead of print 2018/02/13.

22. Simpson, E. R. Sources of estrogen and their importance. J. Steroid Biochem. Mol. Biol. 86, 225-230 (2003). E-pub ahead of print 2003/11/19.

23. Missmer, S. A., Eliassen, A. H., Barbieri, R. L. \& Hankinson, S. E. Endogenous estrogen, androgen, and progesterone concentrations and breast cancer risk among postmenopausal women. J. Natl Cancer Inst. 96, 1856-1865 (2004). E-pub ahead of print 2004/12/17.
24. Carroll, J. S., Hickey, T. E., Tarulli, G. A., Williams, M. \& Tilley, W. D. Deciphering the divergent roles of progestogens in breast cancer. Nat. Rev. Cancer 17, 54-64 (2017). E-pub ahead of print 2016/11/26.

25. Bines, J. et al. Activity of megestrol acetate in postmenopausal women with advanced breast cancer after nonsteroidal aromatase inhibitor failure: a phase II trial. Ann. Oncol. Off. J. Eur. Soc. Med. Oncol. / ESMO 25, 831-836 (2014). E-pub ahead of print 2014/03/13.

26. Espie, M. Megestrol acetate in advanced breast carcinoma. Oncology 51(Suppl 1), 8-12 (1994). E-pub ahead of print 1994/10/01.

27. Birrell, S. N., Roder, D. M., Horsfall, D. J., Bentel, J. M. \& Tilley, W. D. Medroxyprogesterone acetate therapy in advanced breast cancer: the predictive value of androgen receptor expression. J. Clin. Oncol. Off. J. Am. Soc. Clin. Oncol. 13, 1572-1577 (1995). E-pub ahead of print 1995/07/01.

28. A pre-operative window study of Letrozole Plus PR agonist (megestrol acetate) versus letrozole alone in post-menopausal patients with ER-positive breast cancer. https://ClinicalTrials.gov/show/NCT03306472.

29. Centenera M. M., et al. A patient-derived explant (PDE) model of hormonedependent cancer. Mol. Oncol. 12, 1608-1622. 2018; e-pub ahead of print 2018/ $08 / 18$.

30. Soderberg, O. et al. Direct observation of individual endogenous protein complexes in situ by proximity ligation. Nat. Methods 3, 995-1000 (2006). E-pub ahead of print 2006/10/31.

31. Zieba, A., Ponten, F., Uhlen, M. \& Landegren, U. In situ protein detection with enhanced specificity using DNA-conjugated antibodies and proximity ligation. Mod. Pathol. Off. J. US Can. Acad. Pathol. Inc. 31, 253-263 (2018). E-pub ahead of print 2017/09/25.

32. Kornaga, E. N. et al. Evaluation of three commercial progesterone receptor assays in a single tamoxifen-treated breast cancer cohort. Mod. Pathol. Off. J. US Can. Acad. Pathol. Inc. 29, 1492-1500 (2016). E-pub ahead of print 2016/08/ 27.

33. Mohsin, S. K. et al. Progesterone receptor by immunohistochemistry and clinical outcome in breast cancer: a validation study. Mod. Pathol. Off. J. US Can. Acad. Pathol. Inc. 17, 1545-1554 (2004). E-pub ahead of print 2004/07/24.

34. Hammond, M. E. et al. American society of clinical oncology/college of American pathologists guideline recommendations for immunohistochemical testing of estrogen and progesterone receptors in breast cancer (unabridged version). Arch. Pathol. Lab. Med. 134, e48-e72 (2010). E-pub ahead of print 2010/07/01.

35. Edge S. B., American Joint Committee on Cancer. AJCC cancer staging manual, 7th ed. Springer: New York; London, 2010.

36. Torhorst, J. et al. Tissue microarrays for rapid linking of molecular changes to clinical endpoints. Am. J. Pathol. 159, 2249-2256 (2001). E-pub ahead of print 2001/12/06.

37. Allott, E. H. et al. Intratumoral heterogeneity as a source of discordance in breast cancer biomarker classification. Breast Cancer Res.: BCR 18, 68 (2016). E-pub ahead of print 2016/06/29.

38. McShane, L. M. et al. REporting recommendations for tumor MARKer prognostic studies (REMARK). Breast Cancer Res. Treat. 100, 229-235 (2006). E-pub ahead of print 2006/08/26.

39. Mote, P. A. et al. Progesterone receptor A predominance is a discriminator of benefit from endocrine therapy in the ATAC trial. Breast Cancer Res. Treat. 151, 309-318 (2015). E-pub ahead of print 2015/04/29.

40. Privalsky, M. L. The role of corepressors in transcriptional regulation by nuclear hormone receptors. Annu. Rev. Physiol. 66, 315-360 (2004). E-pub ahead of print 2004/02/24.

41. Bulun, S. E. \& Simpson, E. R. Competitive reverse transcription-polymerase chain reaction analysis indicates that levels of aromatase cytochrome P450 transcripts in adipose tissue of buttocks, thighs, and abdomen of women increase with advancing age. J. Clin. Endocrinol. Metab. 78, 428-432 (1994). E-pub ahead of print 1994/02/01.

42. Brown, K. A. et al. Menopause Is a Determinant of Breast Aromatase Expression and Its Associations With BMI, Inflammation, and Systemic Markers. J. Clin. Endocrinol. Metab. 102, 1692-1701 (2017). E-pub ahead of print 2017/03/23.

43. Geisler, J. et al. Letrozole is superior to anastrozole in suppressing breast cancer tissue and plasma estrogen levels. Clin. Cancer Res.: Off. J. Am. Assoc. Cancer Res. 14, 6330-6335 (2008). E-pub ahead of print 2008/10/03.

44. Weiler, P. J. \& Wiebe, J. P. Plasma membrane receptors for the cancer-regulating progesterone metabolites, 5alpha-pregnane-3,20-dione and 3alpha-hydroxy-4pregnen-20-one in MCF-7 breast cancer cells. Biochem. Biophys. Res. Commun. 272, 731-737 (2000). E-pub ahead of print 2000/06/22.

45. Randomized Controlled Trial of Neo-adjuvant Progesterone and Vitamin D3 in Women With Large Operable Breast Cancer and Locally Advanced Breast Cancer. https://ClinicalTrials.gov/show/NCT01608451.

46. Primary Progesterone Therapy for Operable Breast Cancer. https://ClinicalTrials. gov/show/NCT00123669. 
47. Cui, X., Schiff, R., Arpino, G., Osborne, C. K. \& Lee, A. V. Biology of progesterone receptor loss in breast cancer and its implications for endocrine therapy. J. Clin. Oncol. Off. J. Am. Soc. Clin. Oncol. 23, 7721-7735 (2005). E-pub ahead of print 2005/10/20.

48. Winqvist, R. et al. Loss of heterozygosity for chromosome 11 in primary human breast tumors is associated with poor survival after metastasis. Cancer Res. 55, 2660-2664 (1995). E-pub ahead of print 1995/06/15.

49. Tomlinson, I. P., Nicolai, H., Solomon, E. \& Bodmer, W. F. The frequency and mechanism of loss of heterozygosity on chromosome $11 \mathrm{q}$ in breast cancer. $J$. Pathol. 180, 38-43 (1996). E-pub ahead of print 1996/09/01.

50. Stone, A. et al. DNA methylation of oestrogen-regulated enhancers defines endocrine sensitivity in breast cancer. Nat. Commun. 6, 7758 (2015). E-pub ahead of print 2015/07/15.

51. Purdie, C. A. et al. Progesterone receptor expression is an independent prognostic variable in early breast cancer: a population-based study. Br. J. Cancer 110 565-572 (2014). E-pub ahead of print 2013/12/05.

52. Hershman, D. L. et al. Early discontinuation and non-adherence to adjuvant hormonal therapy are associated with increased mortality in women with breast cancer. Breast Cancer Res. Treat. 126, 529-537 (2011). E-pub ahead of print 2010/ 08/31.

53. Endocrine therapy with or without abemaciclib (LY2835219) following surgery in participants with breast cancer. https://ClinicalTrials.gov/show/NCT03155997.
54. Adjuvant ribociclib with endocrine therapy in hormone receptor $+/ \mathrm{HER} 2-$ high risk early breast cancer. https://ClinicalTrials.gov/show/NCT03078751.

55. PALbociclib CoLlaborative Adjuvant Study: a randomized phase III trial of palbociclib with standard adjuvant endocrine therapy versus standard adjuvant endocrine therapy alone for hormone receptor positive $(\mathrm{HR}+)$ human epidermal growth factor receptor 2 (HER2)-negative early breast cancer. https:// ClinicalTrials.gov/show/NCT02513394.

56. Morgan, L. R. Megestrol acetate $\mathrm{v}$ tamoxifen in advanced breast cancer in postmenopausal patients. Semin. Oncol. 12(1Suppl 1), 43-47 (1985). E-pub ahead of print 1985/03/01.

57. Jonat, W. et al. A randomised trial comparing two doses of the new selective aromatase inhibitor anastrozole (Arimidex) with megestrol acetate in postmenopausal patients with advanced breast cancer. Eur. J. Cancer. 32a, 404-412 (1996). E-pub ahead of print 1996/03/01.

58. Buzdar, A. U. et al. A phase III trial comparing anastrozole (1 and 10 milligrams), a potent and selective aromatase inhibitor, with megestrol acetate in postmenopausal women with advanced breast carcinoma. Arimidex Study Group. Cancer 79, 730-739 (1997). E-pub ahead of print 1997/02/15.

59. Buzdar, A. et al. Phase III, multicenter, double-blind, randomized study of letrozole, an aromatase inhibitor, for advanced breast cancer versus megestrol acetate. J. Clin. Oncol. Off. J. Am. Soc. Clin. Oncol. 19, 3357-3366 (2001). E-pub ahead of print 2001/07/17. 